\title{
Optical Fourier transform characterization of fish scale age
}

\author{
Silverio P. Almeida, Roger W. Wygant, Ambrose Jearld, Jr., and Judith A. Penttila
}

\begin{abstract}
Optical Fourier transforms of haddock fish scales were made for fish taken from two different bodies of water. The scales in each group varied in age from 2 to $10 \mathrm{yr}$. A comparison of the image processed digitized Fourier transforms for the scales was made on a personal computer. Statistical correlation studies of these transforms were performed, and the results show a fairly good age discrimination between the scales in each age group.
\end{abstract}

\section{Introduction}

The scales of many species of fish show annular growth rings analogous to the growth rings found in trees. There are also higher frequency structures within these rings. Scale growth occurs around the circumference of the scale leaving the high frequency structure unchanged. Thus, as the scale grows, the frequencies of the small structures change relative to the size of the scale. These changes are manifested by magnifying images of the scales so that they are all the same size. If specific areas of the scale images are Fourier transformed, corresponding areas on different scales should yield corresponding frequency spectra. These spectra may then be compared and grouped according to the age of the fish. However, since growth patterns vary with habitat, it is only possible to compare fish caught in the same area.

Our study used scales from haddock species caught in two different bodies of water, i.e., Brown's Bank and George's Bank. It is not known to us whether there is any exchange of fish between these areas. There were five age groups used from each bank: 2-6 yr olds from Brown's Bank and 3-6 and 10 yr olds from George's Bank. Ninety percent of the fish from Brown's Bank and $86 \%$ from George's Bank were correctly aged.

Silverio Almeida and R. W. Wygant are with Virginia Polytechnic Institute \& State University, Physics Department, Blackburg, Virginia 24061; the other authors are with National Marine Fisheries Services, Northeast Fisheries Center, Woods Hole Laboratory, Woods Hole, Massachusetts 02543.

Received 28 November 1986.

0003-6935/87/122299-07\$02.00/0.

(C) 1987 Optical Society of America.

\section{Theory}

A. Physiology of the Fish Scales

The annular rings of the fish scales are composed of higher frequency, irregularly spaced circuli. During summer months, growth is more rapid, and these circuli are widely spaced. During the winter, growth is slower, and the circuli are closer together, so that there appear alternating regions of high and low circuli density, i.e., the annular rings. There also exist radial structures within the scales, presumably circulatory in nature. ${ }^{1}$ These radial structures have an approximately constant frequency throughout the scale. Once these structures have been formed, they remain essentially unchanged for the life of the fish. Different individuals will have had varying growth rates and thus varying circuli and radial structure frequencies. However, the structure spacing should be in proportion to the growth rate. Also, the size of the scale is proportional to both the growth rate and age of the fish. By appropriately magnifying the image of each scale so that they are all the same size, the problem of varying growth rates within an age group is alleviated, and the images from each age group are given a different magnification [see Fig. 1(a)]. Assuming that the only actual differences in the scale structure frequencies arise from the varying growth rates, the different magnifications given each age group should yield structure frequencies unique to each age group.

\section{B. Derivation of the Digitized Image}

To study these frequency differences, the scale images were Fourier transformed [see Fig. 1(c)]. If the amplitude transmittance in the original scale image transparency is represented by $f(x, y)$ and its Fourier transform is represented by $F\left(k_{x}, k_{y}\right)$, then $F\left(k_{x}, k_{y}\right)$ is given by

$$
F\left(k_{x}, k_{y}\right)=\iint f(x, y) \exp \left[-2 \pi i\left(x k_{x}+y k_{y}\right)\right] d x d y .
$$

This could have been done either optically with a lens 


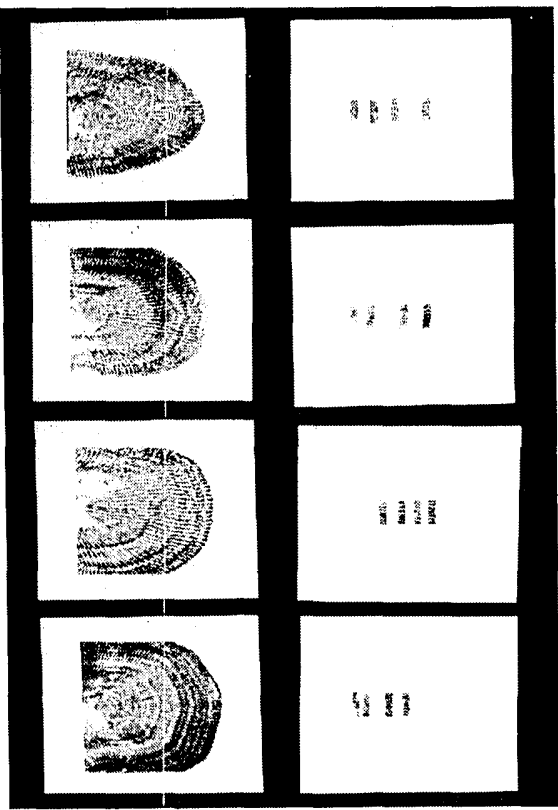

(a)
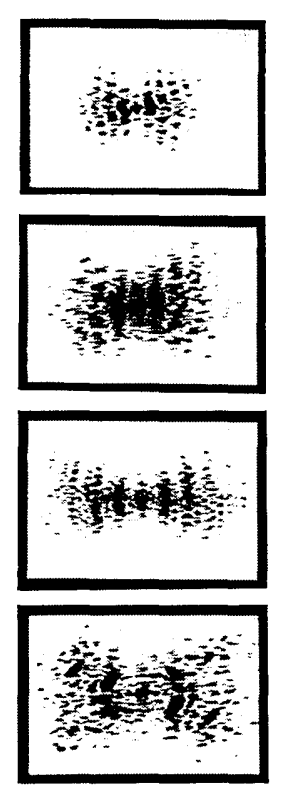

(c)
Fig. 1. (a) Fish scale photographs from George's Bank. Age range from top to bottom 2, 3, 4, 5 yr. (b) Windows showing first year summer and winter circuli (left two) and second year summer and winter circuli (right two). (c) Optical Fourier transforms for the corresponding scales in (a) for the second summer window [i.e., third window from the left in (b)].

or digitally with a computer. We used a Fourier transform lens with the scale image in the front focal plane of the lens, a $2 f$ setup. ${ }^{2,3}$ The use of the coherent optical system is why the amplitude transmittance of the scale is used in Eq. (1). Transforms were made with windows over different summer and winter rings and recorded photographically. Using the definition of the step function,

$$
\theta(x)= \begin{cases}1, & x \geq 0 \\ 0, & x<0,\end{cases}
$$

the window can be represented by

$$
\left[\theta\left(x_{1}\right)-\theta\left(x_{2}\right)\right]\left[\theta\left(y_{1}\right)-\theta\left(y_{2}\right)\right]=\left\{\begin{array}{l}
1, x_{1} \leq \dot{x} \leq x_{2}, y_{1} \leq y \leq y_{2} \\
0, \text { otherwise. }
\end{array}\right.
$$

Incorporating this into Eq. (1), the expression for $F\left(k_{x}, k_{y}\right)$ yields a new function $F^{\prime}\left(k_{x}, k_{y}\right)$ given by

$$
\begin{aligned}
F^{\prime}\left(k_{x}, k_{y}\right)= & \iint f(x, y) \exp \left[-2 \pi i\left(x k_{x}+y k_{y}\right)\right] \\
& \times\left[\theta\left(x_{1}\right)-\theta\left(x_{2}\right)\right]\left[\theta\left(y_{1}\right)-\theta\left(y_{2}\right)\right] d x d y \\
= & \int_{x_{1}}^{x_{2}} \int_{y_{1}}^{y_{2}} f(x, y) \exp \left[-2 \pi i\left(x k_{x}+y k_{y}\right)\right] d x d y .
\end{aligned}
$$

The function $F^{\prime}$ is the complex amplitude of the light field present in the Fourier transform plane of the lens. This field is recorded photographically, and the negative is converted to a positive. This conversion is done using an incoherent optical system. Thus the intensity transmittance of the negative must be used. The amplitude transmittance of the original negative is given by

$$
t\left(k_{x}, k_{y}\right) \propto\left|F^{\prime}\left(k_{x}, k_{y}\right)\right|^{-\gamma_{1}},
$$

where $\gamma_{1}$ is the gamma of the film used. The intensity transmittance of this negative is then

$$
\tau_{1}\left(k_{x}, k_{y}\right) \equiv\left|t\left(k_{x}, k_{y}\right)\right|^{2} \propto\left|F^{\prime}\left(k_{x}, k_{y}\right)\right|^{-2 \gamma_{1}} .
$$

When the positive is made, its intensity transmittance is given by

$$
\tau_{2}\left(k_{x}, k_{y}\right) \propto \tau_{1}\left(k_{x}, k_{y}\right)^{-\gamma_{2}} \propto\left|F^{\prime}\left(k_{x}, k_{y}\right)\right|^{2 \gamma_{1} \gamma_{2}},
$$

where $\gamma_{2}$ is the gamma of the film used to make the positive. The positive is then imaged onto a detector for digitization, the intensity present on the detector will be proportional to $\tau_{2}\left(k_{x}, k_{y}\right)$.

Assuming that the digitization is linear in the intensity, and that for the discrete points $(i, j)$ in the $\left(k_{x}, k_{y}\right)$ plane the digital number $I(i, j)$ is proportional to the integral of the incident intensity in a small region about $(i, j)$, then

$$
I(i, j) \propto \int_{i-\Delta i / 2}^{i+\Delta i / 2} \int_{j-\Delta j / 2}^{j+\Delta j / 2}\left|F^{\prime}\left(k_{x}, k_{y}\right)\right|^{\gamma} d k_{x} d k_{y},
$$

where $\gamma$ is the overall gamma of the photographic procedure, and $(\Delta i, \Delta j)$ is the size of a digital pixel in the $\left(k_{x}, k_{y}\right)$ plane. If Eq. (4) is substituted into Eq. (8), the result is the intensity distribution of the digitized image in terms of the amplitude transmittance of the original fish scale transparency with a window over a selected area:

$$
\begin{aligned}
I(i, j) \propto & \int_{i-\Delta i / 2}^{i+\Delta i / 2} d k_{x} \int_{j-\Delta j / 2}^{j+\Delta j / 2} d k_{y} \\
& \times\left|\int_{x_{i}}^{x_{2}} d x \int_{y_{1}}^{y_{2}} d y f(x, y) \exp \left[-2 \pi i\left(x k_{x}+y k_{y}\right)\right]\right|^{\gamma} .
\end{aligned}
$$

A visual comparison was made after recording the negative of the Fourier transform images, and it was decided to digitize the transforms of the second summer circuli for a quantitative computer-based comparison [see Fig. 1(b)]. The philosophy behind the decision to carry out a computer-based comparison was this. Our laboratory has in the past conducted research on the optical correlation of diatom patterns. One result of this research was the realization that the reliability of the correlations was strongly dependent on the rotational alignment of the patterns being correlated. The computer offers an easy and exactly reproducible method of correcting for these rotational alignment errors. Digital image processing techniques also offer relatively simple methods of dealing with image preprocessing requirements.

\section{Statistical Image Comparison Method}

To obtain a quantitative measure of the similarity of different images, the correlation coefficient was used ${ }^{4}$ :

$$
r=\frac{\sum_{i, j=1}^{N}\left[I_{1}(i, j)-\bar{I}_{1}\right]\left[I_{2}(i, j)-\bar{I}_{2}\right]}{\left.\left\{\sum_{i, j=1}^{N}\left[I_{2}(i, j)-\bar{I}_{1}\right]^{2} \sum_{i, j=1}^{N}\left[I_{2}(i, j)-\bar{I}_{2}\right)^{2}\right]\right\}^{1 / 2}},
$$


where $I_{1}(i, j)$ and $I_{2}(i, j)$ are the intensities of the images being compared at the point $(i, j), N$ is the number of pixels in each row and column of the digitized image, and the $\bar{I}$ terms are the average intensities of the images given by

$$
\vec{I}_{1,2}(i, j)=\left[\sum_{i, j=1}^{N} I_{1,2}(i, j) /\left(\sum_{i, j=1}^{N} 1\right)\right] .
$$

Unfortunately, the indices $i, j$ cannot just run through the elements of an $\mathrm{N} \times \mathrm{N}$ image matrix. The primary reason for this is that it is practically impossible to digitize each image with perfect alignment. If one looks at the Fourier transform of a typical scale pattern [see Fig. 1(c)], it can be seen that the above correlation coefficient would be very sensitive to both rotational and translational alignment errors, which is indeed the case. For this reason, Eq. (10) must be replaced by

$$
r=\frac{\sum_{(i, j) \in R}\left[I_{1}(i, j)-\bar{I}_{1}\right]\left[I_{2}\left(t^{\prime}(i, j)\right)-\bar{I}_{2}\right]}{\left\{\sum_{(i, j) \in R}\left[I_{1}(i, j)-\bar{I}_{1}\right]^{2} \sum_{(i, j) \in R}\left\{I_{2}\left[t^{\prime}(i, j)\right]-\bar{I}_{2}\right\}^{2}\right\}^{1 / 2}},
$$

where $t^{\prime}(i, j)$ represents a rotational and translational coordinate transformation to correct alignment errors, $R$ represents the region of overlap of the two image matrices, and the $\bar{I}$ terms now refer only to the intensities within $R$ :

$$
\bar{I}_{1,2}(i, j)=\left[\sum_{(i, j) \in R} I_{1,2}(i, j)\right] /\left[\sum_{(i, j) \in R} 1\right] .
$$

To accomplish the coordinate transformation, all coordinates are referenced to the center of the Fourier transform patterns, and the rotation is about this center. An attempt was made to define the orientations of the images using the centers of mass and the princi$\mathrm{pal}$ axes of the inertia tensors of the images treating intensity as mass. Unfortunately, this process was not sufficiently accurate, and the orientations eventually used were determined visually with a cursor. The coordinate transformation used was ${ }^{5}$

$$
\begin{aligned}
\left(\begin{array}{l}
x_{2}-x_{2 \mathrm{~cm}} \\
y_{2}-y_{2 \mathrm{~cm}}
\end{array}\right) & =\left(\begin{array}{rr}
\cos \theta & \sin \theta \\
-\sin \theta & \cos \theta
\end{array}\right)\left(\begin{array}{l}
x_{1}-x_{1 \mathrm{~cm}} \\
y_{1}-y_{1 \mathrm{~cm}}
\end{array}\right) \\
& =\left[\begin{array}{ll}
\left(x_{1}-x_{1 \mathrm{~cm}}\right) & \cos \theta+\left(y_{1}-y_{1 \mathrm{~cm}}\right) \sin \theta \\
-\left(x_{1}-x_{1 \mathrm{~cm}}\right) \sin \theta+\left(y_{1}-y_{1 \mathrm{~cm}}\right) \cos \theta
\end{array}\right],
\end{aligned}
$$

which yields

$$
\left(\begin{array}{l}
x_{2} \\
y_{2}
\end{array}\right)=\left(\begin{array}{lr}
\left(x_{1}-x_{1 \mathrm{~cm}}\right) & \cos \theta+\left(y_{1}-y_{1 \mathrm{~cm}}\right) \sin \theta+x_{2 \mathrm{~cm}} \\
-\left(x_{1}-x_{1 \mathrm{~cm}}\right) \sin \theta+\left(y_{1}-y_{1 \mathrm{~cm}}\right) \cos \theta+y_{2 \mathrm{~cm}}
\end{array}\right),
$$

where the $x_{i}$ and $y_{i}$ are pixel coordinates, the $x_{\mathrm{cm}}$ and $y_{\mathrm{cm}}$ terms are the coordinates of the centers of the Fourier transform patterns, and $\theta$ is defined as the angular difference between patterns 1 and 2 .

Referring again to our typical scale transform [see Fig. 1(c)], it can be seen that the frequencies of interest (the bright spots in the image) occupy only a small portion of the image. To accentuate the differences between images, only areas where one or both of the images have bright spots should be correlated. Thus the correlation region $R$ in Eq. (12) is further modified by specifying a threshold value $T$. Only corresponding pixels $(i, j)$ and $t^{\prime}(i, j)$ with one or both intensities greater than or equal to $T$ are included in $R$.

All Fourier transform images possess a central bright spot, commonly referred to as the dc field component. Since this component is present in all the images, including it in the correlation region $R$ adds no information about the similarities of the fish scales in question. Thus a circular region of a specified radius $r$ about the center of the dc component is excluded from $R$.

A final option used in our correlations was to specify a clip level. Any pixel with an intensity greater than the clip level was set to the clip level. This was used to reduce the unevenness of the intensities within the lobes of the Fourier transform patterns.

\section{Statistical Decision Rule}

To identify a particular specimen with a certain age group, it is impractical to correlate its Fourier transform image against those of all the other specimens. For this reason, templates were constructed for each age group by averaging together all the images from fish within the age group. Each individual could then be compared to a small set of templates and identified with the age group whose template it matched best. To form a statistical data base for the decision making process, each specimen was compared to each template. (Note that specimens from the two different bodies of water were treated separately.)

Because of the extreme non-normality of the distribution of the correlation statistic, the Fisher $Z_{f}$ transformation ${ }^{6}$

$$
Z_{f}=\frac{1}{2} \ln \left(\frac{1+r}{1-r}\right)
$$

was applied to the correlation data. The resulting population distribution of $Z_{f}$ is nearly normal. Due to the small sample sizes available to us, the Student's $t$ distribution was used to characterize the samples of the $Z_{f}$ values. The Student's $t$ distribution is given by

$$
Y(t)=\frac{\Gamma\left(\frac{n}{2}\right)\left(1+\frac{t^{2}}{n-1}\right)^{-n / 2}}{\sqrt{\pi(n-1)} \Gamma\left(\frac{n-1}{2}\right)},
$$

where $n$ is the number of values in the sample distribution. The $t$ in Eq. (17) has a mean of zero and a variance of one and is given by

$$
t=\sqrt{n}\left(Z_{f}-\bar{Z}_{f}\right) / s,
$$

where $s$ is the sample standard deviation,

$$
s=\sqrt{\frac{\sum_{i=1}^{n}\left(Z_{f, i}-\bar{Z}_{f}\right)^{2}}{n-1}},
$$

and $\bar{Z}_{f}$ is the sample mean: 


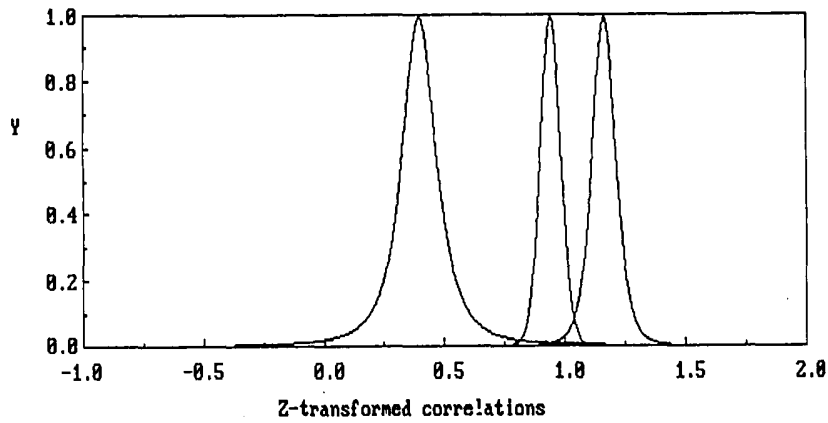

GB4's us. GB4's

$G B 3^{\prime} s, 5^{\prime} s$, and $6^{\prime} s$ us. GB4's

GB10's us. GB4's $n=6$ mean $=1.160$ std. dev. $=.1215$

$n=26$ mean $=.937$ std. dev. $=.2137$

$\mathrm{n}=3$ mean $=.391$ std. dev. $=.1328$

(a)

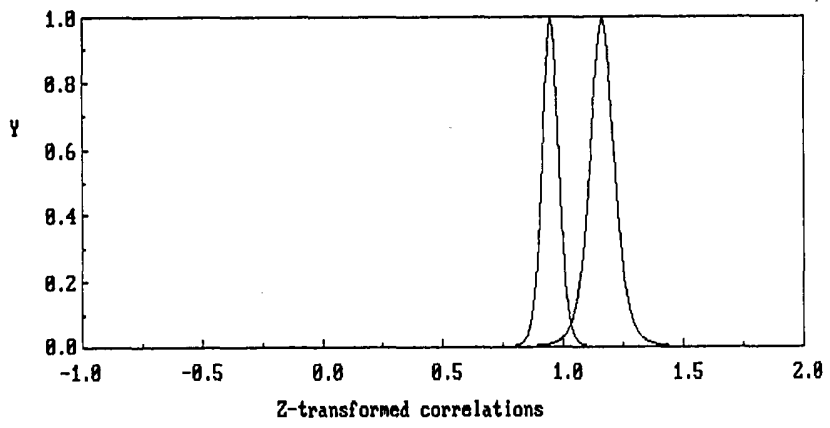

GB4's us. GB4's

GB3's us. GBA's $n=6$ nean $=1.160$ std. dev. $=.1215$

$n=10$ mean $=.943$ std. dev. $=.1856$

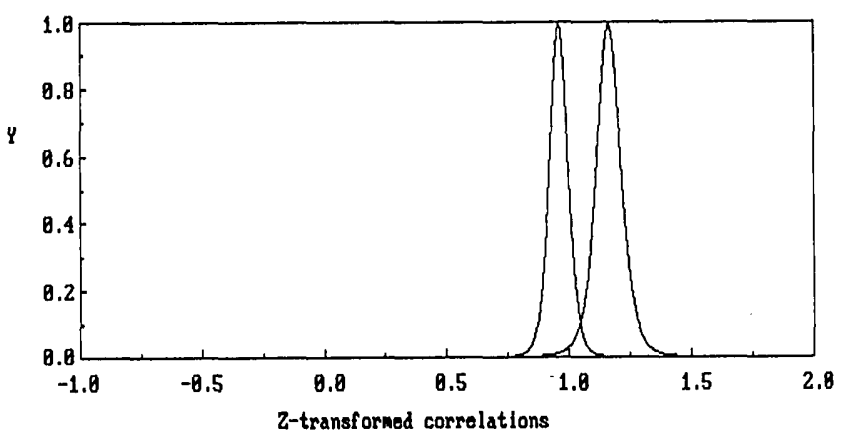

GB4's us. GB4's

GB5's us. GB4's $n=6$ mean $=1.168$ std. dev. $=.1215$

$n=8$ mean $=.954$ std. dev. $=.1857$

(c)

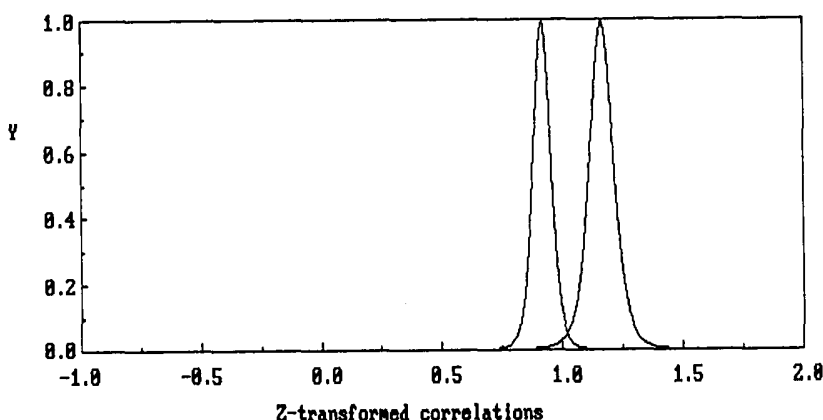

GB4's us. GB4's

GBG's us. GB4's $n=6$ mean $=1.160$ std. dev. $=.1215$
$n=8$ man $=.912$ std. dev. $=.1046$

(d)

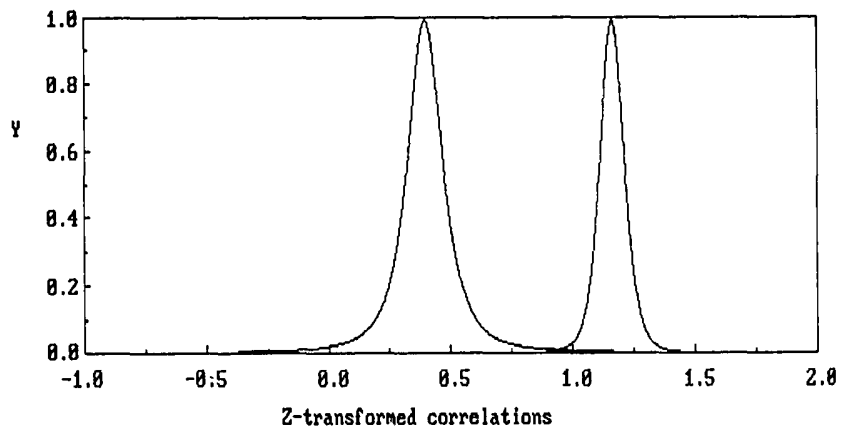

GB4's us. GB4's

$n=6$ mean $=1.160$ std. dev. $=.1215$

GB10's us. GB4's

$n=3$ mean $=.391$ std. dev. $=.1328$

(e)

Fig. 2. Unnormalized $t$ distributions for the George's Bank (GB) scales ranging in ages from 3 to $10 \mathrm{yr}$. Distribution on the right represents the first age given correlated against a Fourier transform (FT) template for its own age group. Hence GB 4-yr vs GB 4-yr FT template. Second curve from the right represents in (a) all the GB 3-, 5-, and 6-yr old Fourier transforms correlated against the GB 4-yr FT template. In (a) the third curve from right correlates the GB 10 yr FTs with the GB 4-yr FT template. 


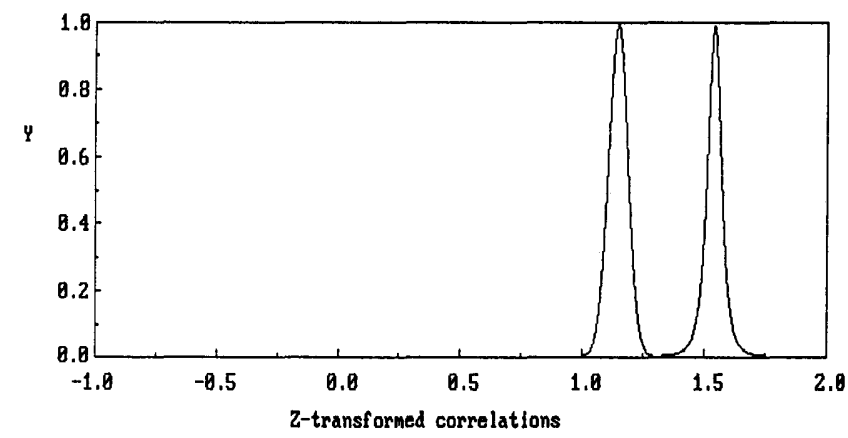

BB4's us. BB4's

$B B 2^{\prime} s, 3^{\prime} s, 5^{\prime} s$, and 6 's us, BB4's

$n=4$ mean $=1.537$

(a)

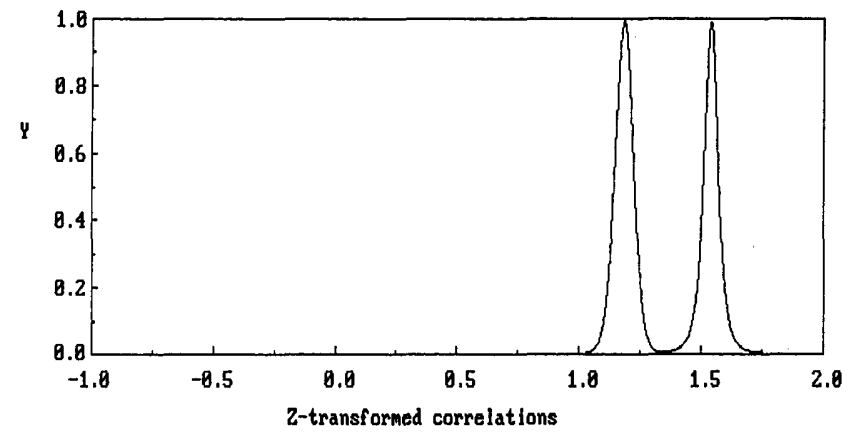

BBA's Us. BB4's

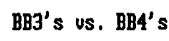

$n=4$ mean $=1.537$ std. dev. $=.0581$

$n=13$ mean $=1.180$ std. dev. $=.1324$

(c)

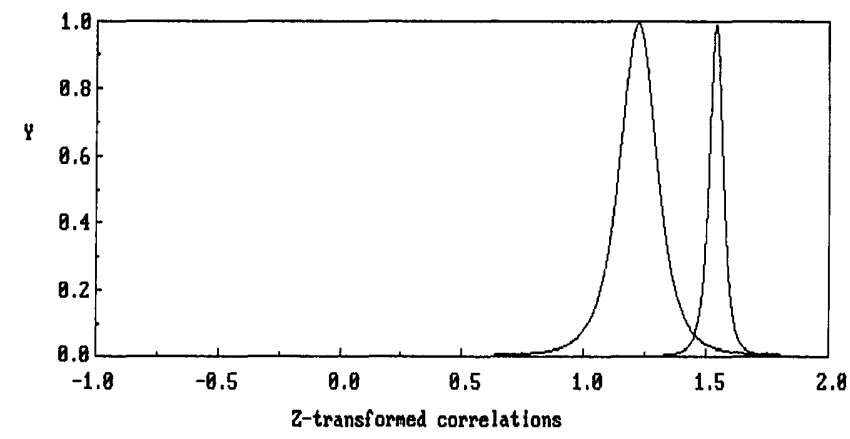

BB4's us. BB4's

$B B 2$ 's us. BB4's $n=4$ mean $=1.537$ std. dev. $=.0581$

$n=4$ mean $=1.216$ std. dev. $=.1592$

(b)

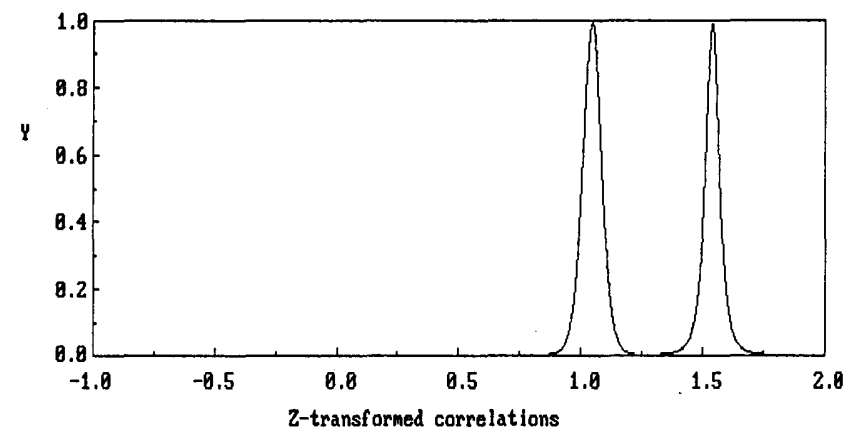

BB4's us. BB4's

BB5's us. BB4's $\mathrm{n}=4$ mean $=1.537$ std. dev. $=.0581$

$\mathrm{n}=9$ mean $=1.041$ std. dev. $=.1128$

(d)

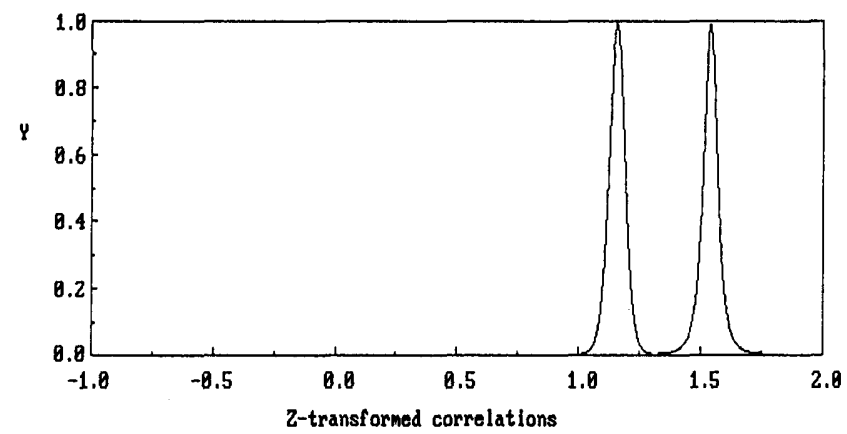

$B B 4^{\prime} s$ us. BB4's

BB6's us. BB4's

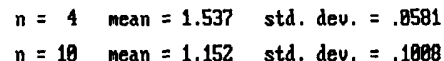

(e)

Fig. 3. Similar distributions to Fig. 2 except these scales are from the Brown's Bank.

$$
\bar{Z}_{f}=\left(\sum_{i=1}^{n} Z_{f, i}\right) /\left(\sum_{i=1}^{n} 1\right) .
$$

Note that $\bar{Z}_{f}, n$, and $s$ refer to particular distributions, for example, the distribution of $Z_{f}$ values of 6 yr vs the template made from 6 yr olds or the distribution of $Z_{f}$ values of all 3,4 , and 5 yr olds vs the template made from 6 yr olds. Figures 2 and 3 show some representative distributions.
The distributions of each age group's correlations against each template were calculated. Also, for each template, the distribution of all specimens not in that template's age group was calculated. To determine whether an individual might belong to a particular age group, the $Z_{f}$ of that individual's correlation with that age group's template was compared to the distribution of individuals in the template's age group as well as to the distribution of individuals not in the template's 


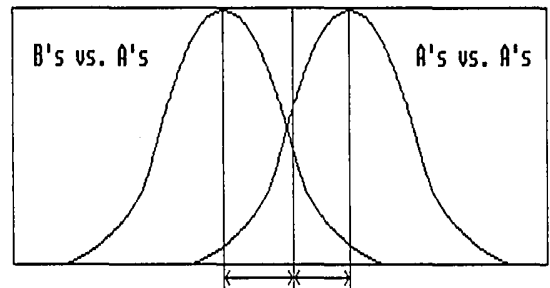

$t(B, A) t(A ; A)$

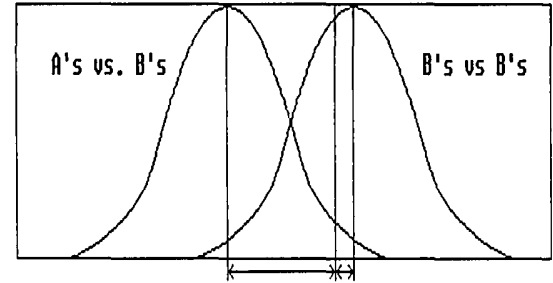

$t(A, B) \quad t(B, B)$

Fig. 4. Decision rule $|t(A, B)|-|t(B, B)|>|t(B, A)|-|t(A, A)|$.

age group. The $t$ statistic of Eq. (18) was used in this comparison. If the specimen's $Z_{f}$ fit better to the distribution of the template's age group, it was considered to be a possible member of that age group. If a specimen fit more than one group, it was compared to the distributions of these age groups against their own and each other's templates. It would then be identified with the age group with which it matched with the least ambiguity. For example, assume that a particular individual happened to match age groups $A$ and $B$. Let us define $t(A, B)$ as the $t$ value [Eq. (18)] of the individual's $Z_{f}$ transformed correlation with the template of age group $B$ against the distribution of the $Z_{f}$ transformed correlations of age group $A$ with the template of age group $B$. The individual would then be identified with age group $B$ if

$$
|t(A, B)|-|t(B, B)|>|t(B, A)|-|t(A, A)| .
$$

Figure 4 provides a graphic illustration of this decision rule.

\section{Experimental Procedure}

Prior to any correlating or template production, all the images were subjected to two processes. First, to subdue electronic digitization noise and coherent speckle noise, each image was averaged with a flat $5 \times 5$ averaging operator. Second, each image had a threshold set at a particular level. This was done because the background level varied widely between images. By selecting a specific threshold for each image, the histograms of all the images could be made similar. The actual algorithm used was to set all pixels with intensities less than the threshold to zero and to stretch the remaining intensity levels to the original 8-bit dynamic range (0-255).

After a final set of templates had been produced, many sets of correlations (every image vs every template) were computed while varying the threshold, clip and dc filter radius in the correlation to determine the optimum parameter set for each template. It was found that the same set of parameters worked best for all the age groups from Brown's Bank, whereas each age group from George's Bank required a different parameter set.

\section{Results and Discussion}

Our final study group consisted of seventy-five specimens, forty from Brown's Bank and thirty-five from George's Bank. Of these, nine were identified with incorrect age groups, four from Brown's Bank and five from George's Bank. The group sizes from Brown's Bank varied between four and thirteen specimens, while those from George's Bank varied between three and ten. Figures 2 and 3 are fairly representative of all the various cross-correlations we calculated for the optical Fourier transforms for both the George and Brown Bank bodies of water. Approximately $90 \%$ of the Brown Bank scales and $86 \%$ of the George Bank scales were correctly aged. Due to the extremely small group sizes and the method of constructing templates from the group and then comparing the group to the template, it seemed imperative to investigate the effects of group size on our results.

The error rate, the mean of the $Z_{f}$ transformed correlation distributions, and the standard deviation of these distributions were compared to the group sizes. It was found that as the group size increased, the means of the distributions tended to decrease very slightly. However, both the error rate and the standard deviations of the distributions did show a significant positive correlation with the group size. The implication of this is that perhaps this method only seems successful because of the extremely small group sizes available for the study. The only way to resolve this is to conduct a similar study with much larger sample sizes. It should be expected that if there is some biological basis for the errors, the error rate should quickly reach some asymptotic value.

\section{Conclusion}

We believe that we have developed a viable method of determining the age of fish based on examination of the fish scales. The possibility exists for converting this method to a highly automated process. However, due to the small sample sizes available to us, we believe there is a need to continue this research with much larger samples. The percentage of poor quality scales misidentified due to structural damage or ambiguous scales due to crossbreeding in the fish would need to be statistically corrected for by visually calibrating scale samples and determining the average number of poor or misidentified scales present in a given body of water. There could also be improvements made to the method, such as computing the Fourier transforms digitally, improving the noise reduction in the images, or improving the template production and correlation procedures.

Direct optical correlation studies using the Fourier transform plane and also the scale image as the sole 
imput are currently under study. A comparison of the three methods is planned.

This work was supported by the U.S. Department of Commerce NOAA contract NA-83-GA-C-0029MOD2.

We also wish to thank L. M. Bernardo and Srisuda Puang-ngern for their laboratory input to this research as well as Kuryan Thomas for his useful discussions on computer problems.

\section{References}

1. W. Harder, Anatomy of Fishes, Part I Text (E. Schweizerbart'sche Verlagsbuchhandlung, Stuttgart, Germany, 1975).

2. J. W. Goodman, Introduction to Fourier Optics (McGraw-Hill, New York, 1968).

3. S. Puang-ngern and S. P. Almeida, Am. J. Phys. 53, 762 (1985).

4. L. Ott, An Introduction to Statistical Methods and Data Analysis (Wadsworth, Belmont, CA, 1977).

5. H. Goldstein, Classical Mechanics (Addison-Wesley, Reading, MA, 1980).

6. A. C. Aitken, Statistical Mathematics (Oliver and Boyd, Edinburgh, England, 1962).
Patter continued from page 2292

latitude in the Sargasso Sea, and one at a high latitude in the Indian Ocean between Madagascar and Antarctica. Comparisons of the flux measurements of the the three quiet seas are drawn, and the results are discussed and analyzed.

Microwave measurements can be used to deduce the thermal properties of the sea surface. They are not seriously degraded by tenuous and moderately dense clouds. However, raining clouds and clouds that contain water droplets do attenuate microwaves significantly. A further advantage of microwave sensing is that the polarities of sea reflections may be discerned. Differences and ratios of polarized signals may be formed to provide information on important sea properties. For example, the difference between horizontally and vertically polarized fluxes can be used to infer sea roughness and the presence of such pollutants as oil films.

The plots taken from the measurements show the following: the effects of the relative, on-axis gain of the collecting aperture for each frequency $(6.6,10.69,18,21$, and $37 \mathrm{GHz})$; the effects of polarization rotation in the outputs of the receiver when the collecting aperture mechanically rotates about a fixed feed; the difference between the flux magnitudes for the horizontal and vertical channels at each of the five frequencies, and for each pointing position, over a $44^{\circ} \mathrm{scan}$ angle; and the rms value of the clutter, as reckoned over the interval of a full swath and derived from the standard error of estimate of the plotted swath response for each channel.

The expected value of the background temperature is computed for each of the three quiet seas that was observed. The background temperature includes contributions from the cosmic background, the downwelling path, the sea surface, and the upwelling path.

This work was done by Joseph M. Stacey of Caltech for NASA's Jet Propulsion Laboratory. Further information may be found in NASA CR-176199 [N85-35322/NSP], "Microwave Properties of a Quiet Sea." Copies may be purchased [prepayment required] from the National Technical Information Service, Springfield, VA 22161, 703-487-4650. Refer to NPO-16691.

\section{CMOS clock synchronizer}

A simple circuit develops a clock signal synchronized with an external gate signal, with a maximum skew of one-quarter of a clock cycle; yet it requires a synchronizing square wave of only twice the designated clock frequency. To effect the same maximum skew, previous circuits required synchronizing signals four times the clock frequency. The relatively low synchronizing frequency of the new circuit is particularly advantageous in radiation environments where the maximum clocking frequency of complementary metal oxide/ semiconductor (CMOS) circuits must be severely derated.

The circuit was originally developed for use in a dual-slope analogto-digital converter. In such a converter, if the clock signal is simply gated without synchronization, the skew between the gate and clock signals can result in up to a 1-bit error in the converter output. With a one-quarter-cycle synchronization, the error from this source is reduced to one-quarter of a bit or less, provided that clock symmetry is good.

The new circuit (see Fig. 5) achieves its low skew by synchronizing an output clock with either the leading or the trailing edge of an input clock-signal pulse, whichever occurs first after the gate signal: the input clock signal is applied directly to flip-flop $F F_{1}$ and, via an inverter, to $F F_{2}$. Thus either $F F_{1}$ is set by the next active edge of the input clock or $\mathrm{FF}_{2}$ is set by the complement of the edge, whichever occurs first. The next active edge occurs within a one-half cycle of the synchronizing signal, which is one-quarter cycle of the designat-

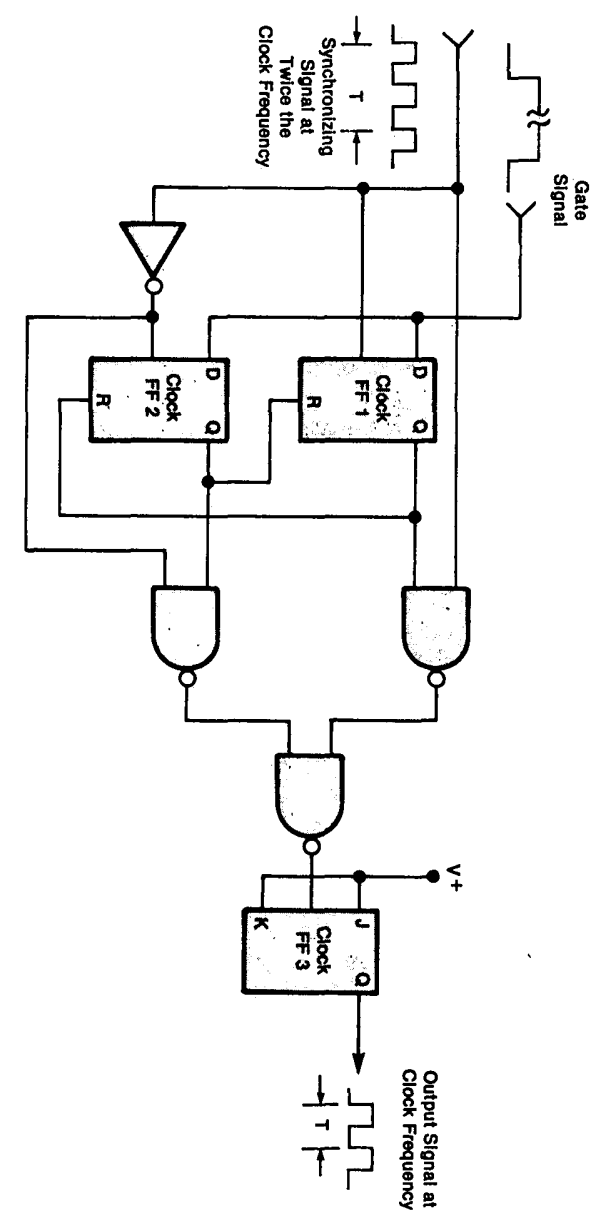

Fig. 5. Clock synchronizer with one-quarter-cycle skew can be constructed from three flip-flops, three NAND gates, and an inverter. In addition to the gate signal to which the clock is to be synchronized, the circuit requires a square-wave input at twice the desired clock frequency.

continued on page 2333 